\title{
Effect of Electroacupuncture on Anovulation, Epinephrine and Norepinephrine Levels in Drug Induced Polycystic Ovary Rats
}

\author{
Mukilan Ramadoss ${ }^{1}$, Gunasekaran Ramanathan ${ }^{2}$ \\ ${ }^{1}$ Senior Lecturer, Department of Physiology, CSI College of Dental Sciences and Research, Madurai, India \\ ${ }^{2}$ Professor, Department of Physiology, VMKV Medical College, Salem, India
}

\begin{abstract}
Background: Earlier studies on the effect of constant electro-acupuncture (EA) treatments in rats with drug-induced polycystic ovary syndrome (PCOS), has been shown to revise the hormones and neurotransmitter levels. Aim: The present study was conducted with the aim to test the hypothesis that repeated EA treatment modulates Epinephrine (EPI) and Norepinephrine (NE) level of PCOS and anovulation. Method: This was done by analyzing EPI and NE level using high-performance liquid chromatography (HPLC) in plasma of normal rats (n=10), drug Estradiol-Valerate $(E V)$ induced PCOS rats $(n=10)$ and PCOS rats treated with EA $(n=10)$ and modulation of ovulation was studied by staining the ovary. Result: The present study shows the concentration of EPI and NE level in the plasma was significantly $(P<0.001)$ lower and reversal of ovulation from anovulation in the PCOS rats treated with $E A$ compared with the EA untreated PCOS rats. Conclusion: The EA treatment reverses the EPI and NE level indicate that PCOS modulates the sympathetic activity of rats and the EA treatment could bring back the Neuroendocrinological state and the sympathetic activity of PCOS rats which turn induce the ovulation back to normal.
\end{abstract}

Keywords: Sympathetic activity, Ovulation, Neuroendocrine, epinephrine, norepinephrine, polycystic ovary

\section{Introduction}

Polycystic ovary syndrome (PCOS), is a complex endocrine and metabolic disorder (1) It causes anovulation in the reproductive existence of females and in many cases it leads to infertility. Earlier studies have suggested the pathogenesis of PCOS, but still, its underlying mechanism for the PCOS is unclear and inconsistent. Untreated PCOS leads to the risk of cancer, diabetes, etc. (2), and many risk factors are associated with PCOS such as increased prevalence of dyslipidemia, (3) hypertension (4) and CVDs (5). The most common features of PCOS, like central obesity, hyperinsulinaemia (6), hypertension and obstructive sleep apnoea (OSA), are associated with the chronic sympathetic nervous system overactivity $(7,8)$ increase in the catecholamine (9), and nerve growth factor levels (10) leading to speculation that the sympathetic nervous system may be implicated in the pathogenesis of this condition.

In most of the PCOS cases, pharmacological treatment for anovulation is ineffective or it has lots of side effects like superovulation or improper ovulation. Many studies suggest that beyond the pharmacological treatment repeated EA treatment may reverse the anovulation successful (11) EA treatment is a safe and effective treatment for woman with PCOS and may also have a role in effectively increasing the blood flow to the ovaries, shrinking of ovarian volume and the number of ovarian cysts, it enhances the insulin sensitivity, decreases blood glucose levels and reverses the insulin levels and sympathetic activity to normal (11). However, well- planned, randomized controlled trials are needed to elucidate the true effect of EA treatment by studying the altered sympathetic activity and catecholamine levels in PCOS.
The mechanisms behind the beneficial effect of EA on PCOS in animal model studies are not clear, particularly its involvement in the nervous system. Currently, the studies on the sympathetic nervous system may offer a new therapeutic target in PCOSt \& this is the part of published study (12), the present study was aimed to test the hypothesis that modulation of anovulation and sympathetic nerve activity by repeated EA treatment in steroid PCOS rats. This was done by analyzing EPI and NE level of plasma and by studying the histopathology of the ovary was done after repeated EA treatment on drug-induced PCO rats.

\section{Materials and Method}

This in vivo animal experimental study was carried out at $\mathrm{KM}$ college of pharmacy, for a period of 3 months, in the year 2015. All methods were carried out in accordance with the approved guidelines for the VMKV medical university Institutional animal ethical board, Wister albino rats 30 adult virgin cyclic rats weighing 200-250g were housed in cages at a controlled temperature, for a period of one week 12-12 hours a day, night cycle is maintained and has free access to food and ad libitum water.

PCO initiation: After acclimatization the rats were divided into three groups, control $(n=10), \operatorname{PCOS}(n=10)$ and PCOS + EA $(n=10)$. For PCO initiation each normal female rat $(n-$ 10) was injected with single I.m injection of $4.5 \mathrm{mg}$ of EV (Progynon deport, German Remedies). The animals housed and were handled according to CPCSEA guidelines.

PCO Confirmation: After one month in all the control and experimental group rats vaginal smear was taken daily between 9 am to 11 am. The EV-induced rats show prolonged diestrus stage when compared to control that we consider it as PCOS group. In the vaginal smear, the

Volume 6 Issue 1, January 2017

www.ijsr.net

Licensed Under Creative Commons Attribution CC BY 


\section{International Journal of Science and Research (IJSR) \\ ISSN (Online): 2319-7064}

Index Copernicus Value (2015): 78.96 | Impact Factor (2015): 6.391

prolonged diestrus was the conformance test for PCOS appearance. The smears were stained using crystal violet (NICE chemicals) and viewed under a microscope and the predominant cell type in a vaginal smear in the PCOS is leukocytes the main cell type in diestrus stage.

\section{Electroacupuncture (EA) treatment:}

Repeated low-frequency EA treatment was carried out in the PCOS+EA group, each treatment lasted for 20 minutes for everyone in two days up to 8-10 wks. The rats were anesthetized and suspended in a harness. The needles were inserted bilaterally in the biceps femoris and erector spinae muscle in the somatic segment corresponding to the innervations of the ovaries. The needles were inserted to the depth of $0.3-0.5 \mathrm{~cm}$ and then connected to an electrical stimulator with the low frequency of $2 \mathrm{~Hz}$ and the burst frequency of $80 \mathrm{~Hz}$. The intensity was adjusted until the local muscle contraction valid from $0.8-1.3 \mathrm{~mA}$. After the treatment period, the animals in the control group, PCOS group, and the PCOS+EA treatment group were used for the EPI and NE estimation.

\section{Blood samples}

After the experimental period, animals were anesthetized with sodium pentobarbital and the blood samples were collected in flasks with heparin by means of catheterization of the abdominal aorta of the control and PCOS rats were collected before and after EA treatment without any preservatives $(13,14)$ and transported at $4^{\circ} \mathrm{C}$ for estimation of EPI and, NE Plasma was separated immediately by centrifugation, at 2000 R.P.M for five minutes at $2{ }^{\circ} \mathrm{C}$ and stored at $-30^{\circ} \mathrm{C}$. Plasma EPI and NE was estimated within 60 days of storing at $-30^{\circ} \mathrm{C}$.

\section{HPLC analysis:}

To measure plasma EPI and NE levels high-performance liquid chromatography (HPLC) with electrochemical detection method was used $(15,16,17)$

\section{Histological study of ovary}

The normal control and at the end of all experimental groups were euthanized, ovaries were removed, cleaned of adherent connective fat tissue, and fixed in $4 \%$ formaldehyde buffer; tissue samples were prepared wax blocks and sections were taken using microtome. Tissue sections were stained with haematoxylin-eosin (NICE chemicals), the slides were viewed under a light microscope and a well-trained pathologist performed the follicle population, morphological variation and number of the follicle. If an ovary is polycystic the follicles are cystic in appearance, follicles become atretic and hyperplasia of stoma was observed, otherwise, they were considered as healthy.

\section{Statistical analyses:}

All statistical analysis were performed with SPSS 11.0 software. EPI and NE data were analyzed using one-way ANOVA.

\section{Result}

\section{Vaginal smear analysis}

In a regular cyclic estrous cycle in rats show four phases estrus, metestrus, diestrus and preposterous. The stages of the cycle can be determined by the particular cell type appearance in the vaginal smear Estrous stage: clumps of large cornified cells. Metestrous: nonnucleated epithelial cells with a large number of leucocytes. Diestrous stage: predominantly leucocytes with the scanty amount of epithelial and cornified cells. Proestrus stage: mostly rounded epithelial cells but few cells showing cornification showing early stages of estrous

Vaginal smear analysis shows the stages of estrous cycle. Normal control group appears with regular cyclicity with Estrous, Metestrous, Diestrous and Proestrous. PCOS group appears as prolonged diestrus stage ( acyclicity) and PCOS + EA group appears with regular cyclicity

\section{Histopathology study of Ovary}

Rats injected with the same dose of EV and killed on day 30 showed a progressive decrease in the number of primary and secondary follicles but it was on day 60 ( Figure /Table 1 ) that the true cystic follicles appeared, follicle morphological variation, follicles become atretic and hyperplasia of stroma were observed. It reveals a well-defined PCOS was fully developed (B) in accordance with previous reports by (18). The present study, after repeated treatment with EA, for 60 days showed normal follicles rapper, the number of cysts was very minimal (C) No substantial morphological differences were found between the EA-treated and the control group. ( A)

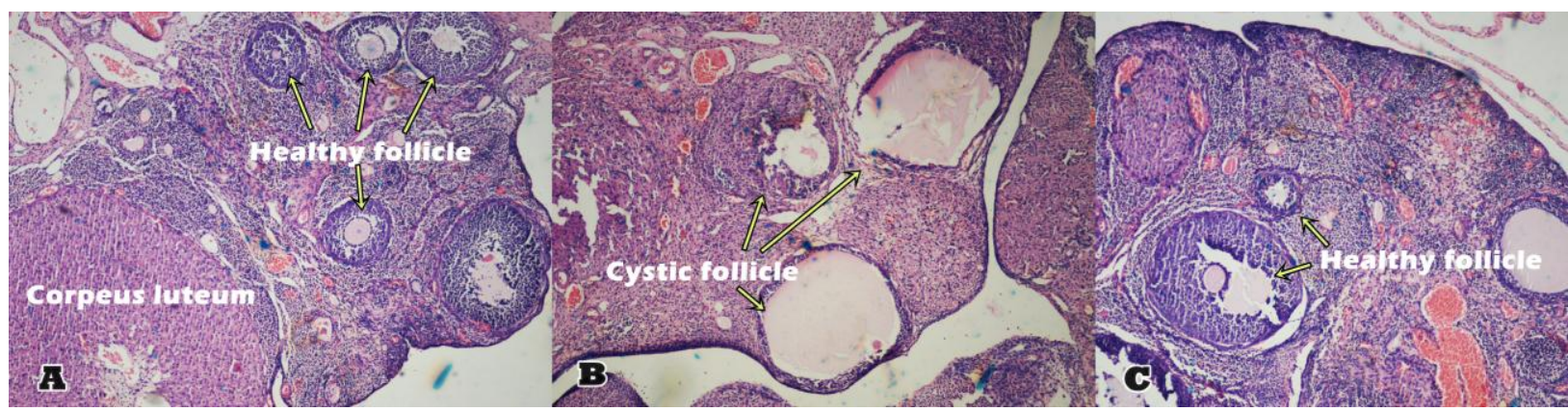

Figure/ Table 1: Histopathologic study of the Ovary

[Figure /Table 1:] Haematoxylin and Eosin stained ovary section. A. The normal control rats ovary with healthy follicle and corpus luteum, B. EV-induced rats ovary shows cystic appearance and C. After electroacupuncture treatment in PCOS rats showing reappearance of healthy follicle 


\section{International Journal of Science and Research (IJSR) \\ ISSN (Online): 2319-7064}

Index Copernicus Value (2015): 78.96 | Impact Factor (2015): 6.391

Epinephrine and Norepinephrine level in rat plasma

Figure / Table 2: Epinephrine and Norepinephrine level in rat plasma (Normal and PCO, EAT) (HPLC)

\begin{tabular}{|c|c|c|c|c|}
\hline & $\begin{array}{c}\text { Normal control ng/ml } \\
\text { Mean }+ \text { SD }\end{array}$ & $\begin{array}{c}\text { PCOS -Before EAT ng/ml } \\
\text { Mean +SD }\end{array}$ & $\begin{array}{c}\text { PCOS- After EAT ng/ml } \\
\text { Mean +SD }\end{array}$ & P value \\
\hline Epinephrine & $0.256+0.04902$ & $0.41190+.03925$ & $0.26000+.05199$ & $\mathrm{P}<0.001$ \\
\hline Nor epinephrine & $0.2389+0.05799$ & $0.3994+0.05428$ & $0.25820+0.04944$ & $\mathrm{P}<0.001$ \\
\hline
\end{tabular}

Significant P<0.001 PCOS - Polycystic Ovary Syndrome, EAT: Electro-acupuncture treatment

One-way ANOVA comparison analysis showed in (Figure/Table. 2) the plasma EPI and NE level of Control, PCOS rats before and after EA treatment revealed that EPI and NE levels are increased significantly $(\mathrm{P}<0.001)$ after EA treatment group when compared to PCOS alone. The EA treated PCOS group when it compared to control group the EPI and NE level significantly $(\mathrm{P}<0.001)$ back to normal.

\section{Discussion}

In this modern world women with a sedentary lifestyle and unhealthy food habits may develop PCOS, it is increasing in worldwide in an alarming rate, which is associated with insulin resistance, hyperandrogenism, and obesity. Middleaged women with PCOS showed excitation in sympathetic activity and altered autonomic dysfunction (19) which has a high risk of cardiovascular disease (20). Even though various therapeutic drugs are available to treat PCOS, they all have their own limitation and side effects. EA has shown to be effective in the treatment of PCOS without any side effects.

In the present study rats injected with EV showed polycystic changes through persisting anovulation and significantly high concentration of plasma EPI and NE. It agrees with the previous study that PCOS rat's hypothalamus release increased NE $(9,21,10)$. Our test result shows that repeated EA treatment modulates anovulation and EPI and NE level in rats with steroid-induced PCOS and the elevated EPI and NE concentration in PCOS rats significantly reduce back to normal. Similar results were also observed in $(22,23)$ it indicates increased secretion of catecholamines in PCOS induced hyperactivity of the sympathetic nervous system. In the present study, the follicles in the ovary appeared back to be normal after EA treatment. The number of cystic follicles was decreased it leads to reversal of normal ovulation in rats with PCOS, It agrees with the previous study reported that low-frequency EA treatment induced ovulation in women with PCOS (11).

In PCOS rats attainable stress, the stress response mediated through the sympathetic adrenal medullary axis (SAM) and the release of CRH are increased with increased activity of the HPA axis, which causes increased secretion of the ACTH (24) and leads to glucocorticoid release from the adrenal cortex. The HPA axis along with intra-adrenal mechanisms which involve the adrenal medulla may also regulate the synthesis of steroid hormone from the adrenal cortex. Moreover, hormones from adrenal cortex may influence the adrenomedullary chromaffin cells and vice versa (25). Activation of the SAM axis, however, increases the release of EPI and NE (26). That EA treatment may decrease hyperactivity in the ovarian peripheral sympathetic nerve fibers is in agreement with the theory that EA could modulate sensory, motor, and autonomic outflow at the segmental level (27).

\section{Limitations}

This study on electroacupuncture treatment in drug-induced PCOS was targeted in alteration of sympathetic nervous system activity in a rodent model. Duration of this study was short and small sample size were the limitations of this study. Future study can be carried out in humans in a larger group as a long-term study.

\section{Conclusion}

Repeated EA treatments significantly decrease plasma concentrations of EN and NEN level and reverse the anovulation. This result supports our previous finding the EA treatment revise the increased HR in PCOS rats, it indicate that EA treatment reduce the sympathetic nerve activity However, it remains to be elucidated whether these effects of repeated EA treatments are a result of a direct action on the ovary, a decrease in the sympathetic outflow to the ovary, or both.

\section{Acknowledgements}

We wish to thank, Dr. S. Angelie Jessica, Dr. S. Saravanakumar., and Dr. N. Gururaj, CSI College of Dental Sciences and Research, Madurai, for their animal dissection, staining slides and valuable help for taking photomicroscopic images.

\section{References}

[1] Franks S. Polycystic ovary syndrome. $N$ Engl J Med. 1995 Sep 28;333 (13):853-61

[2] Lakhani K, Prelevic GM, Seifalian AM, Atiomo WU, Hardiman P. Polycystic ovary syndrome, diabetes and cardiovascular disease: risks and risk factors. J Obstet Gynaecol. 2004 Sep;24 (6):613-21.

[3] Talbott, E., Guzick, D., Clerici, A, Sarah B, Katherine D, Karl W, Lewis K. Coronary heart disease risk factors in women with polycystic ovary syndrome. Arteriosclerosis, Thrombosis, and Vascular Biolog. 1995 July; (7) 15, 821-826.

[4] Sampson, M., Kong, C., Patel, A, Unwin R, Jacobs HS. Ambulatory blood pressure profiles and plasminogen activator inhibitor (PAI-1) activity in lean women with and without the polycystic ovary syndrome. Clinical Endocrinology (Oxford). 1996: 45, (5) 623-629.

[5] Paradisi, G., Steinberg HO, Hempfling A, Cronin J, Hook G, Shepard MK, Baron AD. Polycystic ovary

\section{Volume 6 Issue 1, January 2017 www.ijsr.net}




\section{International Journal of Science and Research (IJSR) \\ ISSN (Online): 2319-7064 \\ Index Copernicus Value (2015): 78.96 | Impact Factor (2015): 6.391}

syndrome is associated with endothelial dysfunction. Circulation, 2001 Mar 13;103 (10):1410-5

[6] Dunaif, A., Segal, K.R., Futterweit, W. and Dobrjansky, A. Profound peripheral insulin resistance, independent of obesity, in polycystic ovary syndrome. Diabetes. 1989 Sep;38 (9):1165-74

[7] Lara HE, Ferruz JL, Luza S, Bustamante DA, Borges Y and Ojeda SR. Activation of ovarian sympathetic nerves in polycystic ovary syndrome. Endocrinology, 1993 Dec;133 (6):2690-5.

[8] Schuster A, Farookhi R and Brawer JR. A polycystic ovarian condition in estradiol valerate-treated rats: Spontaneous changes in characteristic endocrine features Biol Reprod. 1984 Oct;31 (3):587-93.

[9] Luza SM, Lizama L, Burgos RA, Lara HE. Hypothalamic changes in norepinephrine release in rats with estradiol valerate induced polycystic ovaries. Biol Reprod. 1995 Feb;52 (2):398-404.

[10]Lara HE, Dissen GA, Leyton V, Paredes A, Fuenzalida $\mathrm{H}$, Fiedler JL, Ojeda SR. An increased intraovarian synthesis of nerve growth factor and its low-affinity receptor is a principal component of the steroid-induced polycystic ovary in the rat. Endocrinology. 2000 Mar;141(3):1059-72

[11] Steiner-Victorin E, Waldenström U, Tägnfors U, Lundeberg T, Lindstedt G, Janson PO. Effects of electro-acupuncture on anovulation in women with polycystic ovary syndrome. Acta Obstet Gynecol Scand. 2000 Mar;79(3):180-8

[12] Mukilan Ramadoss, Gunasekaran Ramanathan, Angelie Jessica Subbiah, Chidambaranathan Natrajan Heart Rate Changes in Electroacupuncture Treated Polycystic Ovary in Rats. J Clin Diagn Res. 2016 Mar; 10(3): CF01-CF03

[13] Boomsma F, Alberts G, Van Eijk L, Man in 't Veld AJ, Schalekamp MA. Optimal collection and storage conditions for catecholamine measurements in human plasma and urine. Clin Chem. 1993 Dec;39(12):2503-8.

[14]DeAlsandro M, Reed $H$, Robertson R, Lewis $S$. Simplified methods of collecting and processing whole blood for quantitation of plasma catecholamines. Laboratory medicine, Vol. 21, No. I, January 1990.

[15] Tsunoda M. Recent advances in methods for the analysis of catecholamines \& their metabolites. Anal Bioanal Chem. 2006 Oct;386(3):506-14.

[16]Peaston RT, Weinkove C. Measurement of catecholamines and their metabolites. Ann Clin Biochem. 2004 Jan;41(Pt 1):17-38

[17] Tareke E, Bowyer JF, Doerge DR. Quantification of rat brain neurotransmitters and metabolites using liquid chromatography/electrospray tandem mass spectrometry and comparison with liquid chromatography/electrochemical detection. Rapid Commun Mass Spectrom. 2007;21(23):3898-904.

[18] Brawer JR, Munoz M, Farookhi R. Development of the polycystic ovarian condition (PCO) in the estradiol valerate treated rat. Biol Reprod. 1986 Oct;35(3):647-55

[19]Zainab Hasan Hashim, Farqad Bader Hamdan, and Anam Rashid Al-Salihi, Autonomic dysfunction in women with polycystic ovary syndrome, Iran J Reprod Med. 2015 Jan; 13(1): 27-34.

[20] Dokras A, Cardiovascular disease risk in women with PCOS. Steroids. 2013 Aug;78 (8):773-6.
[21] Brawer JR, Naftolin F, Martin J and Sonnenschein C. Effects of a single injection of estradiol valerate on the hypothalamic arcuate nucleus and on reproductive function in the female rat. Endocrinology. 1978 Aug;103(2):501-12.

[22] Garcia-Ruiz C, Armando I, Levin G, Escobar ME and Barontini M. Peripheral catecholamine alterations in adolescents with polycystic ovary syndrome. Clin Endocrinol (Oxf). 1998 Aug;49(2):221-8.

[23] Shoupe D and Lobo RA. Evidence for altered catecholamine metabolism in polycystic ovary syndrome. Am J Obstet Gynecol. 1984 Nov 1;150: 56671

[24] Stener-Victorin E, Lundeberg T, Waldenstrom U, Bileviciute-Ljungar I, Janson PO: Effects of electroacupuncture on corticotropin-releasing factor in rats with experimentally induced polycystic ovaries. Neuropeptides. 2001 Oct-Dec;35(5-6):227-31

[25] Bornstein SR, Ehrhart-Bornstein M: Basic and clinical aspects of intrarenal regulation of steroidogenesis. $Z$ Rheumatol. 2000;59 Suppl 2: II/12-7

[26] Chrouses GP: The role of stress and the hypothalamicpituitary- adrenal axis in the pathogenesis of the metabolic syndrome: neuroendocrine and target tissuerelated causes. Int J Obes Relat Metab Disord. 2000 Jun;24 Suppl 2: S50-5.

[27] Andersson S, Lundeberg T. Acupuncture--from empiricism to science: functional background to acupuncture effects in pain and disease. Med Hypotheses. 1995 Sep;45(3):271-81. 